\title{
The effects of compound stimulus preexposure of two elements differing in salience on the acquisition of conditioned suppression
}

\author{
R. E. LUBOW, M. WAGNER, and I. WEINER \\ Tel-Aviv University, Ramat Aviv, Israel
}

\begin{abstract}
Conditioned attention theory (CAT) of latent inhibition (LI) states that parallel learning processes occur during reinforced and nonreinforced stimulus presentation. The present experiments investigated the effects of nonreinforced preexposure of either a compound CS or elements of that compound which differed in salience. Three predictions were advanced: (1) Both the compound and its elements will show an increase in LI as a function of the number of preexposures; (2) the two elements will show different levels of LI, with more LI accruing to the more salient element; (3) overshadowing will occur during compound preexposure. Two experiments, using rats as subjects and a conditioned suppression test, are reported. In Experiment 1 , groups received $0,20,40$, or 80 nonreinforced preexposures to a compound whose elements differed in salience. The results of the subsequent test confirmed predictions 1 and 2. Experiment 2, in which groups were preexposed to either the elements or the compound, provided evidence for an overshadowing effect, confirming prediction 3 from CAT.
\end{abstract}

A review of the latent inhibition (LI) literature led to the conclusion that available explanations of the decremental effects of preexposure of the to-beconditioned stimulus upon subsequent learning were inadequate (Lubow, 1973). The explanations surveyed included habituation of the orienting response (e.g., Wolf \& Maltzman, 1968), selective filter biasing (e.g., Schnur, 1971), competing response (Lubow \& Moore, 1959), complementing response (Lubow, Markman, \& Allen, 1968), conditioned inhibition (Reiss \& Wagner, 1972; Rescorla, 1971), and stimulus salience reduction (Reiss \& Wagner, 1972; Rescorla, 1971).

More recently, four new theories have been proposed that relate to the LI phenomenon: learned irrelevance (Mackintosh, 1973), an information processing model based on priming of short-term memory (Wagner, 1976), associability loss as a function of accurately predicted consequences (Pearce \& Hall, 1980), and a conditioned attention theory (CAT) of preexposure effects (Lubow, Weiner, \& Schnur, 1981). Tests of the latter theory provide the focus for the present paper.

The conditioned attention theory is based on the use of attention as a hypothetical construct with the

This research, part of the second author's master's thesis, was supported by a grant from the Ford Foundation received through the Israel Foundations Trustees. Requests for reprints should be sent to R. E. Lubow, Department of Psychology, Tel-Aviv University, Ramat-Aviv 69978, Israel. The paper was prepared while the first author was a visiting fellow at the Department of Psychology, Yale University. The authors thank Paul Schnur and Allan Wagner for their comments on an earlier draft of the paper. characteristics of a Pavlovian response. The attentional response $\left(R_{A}\right)$ is postulated to be conditionable, but to differ from a CR in the typical Pavlovian paradigm in that the CS is assumed to elicit $R_{A}$ on first presentation. In a Pavlovian conditioning procedure, the CS typically does not initially elicit the response that is to be conditioned but comes to do so after training. CAT assumes that the attentional response to a stimulus is elicited on first presentation, and the function of a consequent US is to prevent that $\mathrm{R}_{\mathrm{A}}$ from diminishing. It is the maintenance of the $R_{A}$ that is accomplished by the usual conditioning procedures. Therefore, when $R_{A}$ is maintained over repeated trials with one stimulus, as a result of pairing that stimulus with a second stimulus, $\mathbf{R}_{\mathbf{A}}$ should be considered as a conditioned attentional response, $C R_{A}$.

It is further proposed that, in the absence of a significant event (such as US) following the stimulus, a parallel learning process takes place which may be conceived of as the conditioning of inattention to that stimulus. Mackintosh (1975) has proposed the term "learning to ignore" to describe reduced associability as a result of such stimulus exposure experiences. However, it is not sufficient to argue that stimuli uncorrelated with reinforcement lose their attention-eliciting properties or that the organism learns to ignore such stimuli; the mechanism of such a process must be specified. A similar conclusion has been reached by Rescorla and Wagner (1972, p. 94) and Mackintosh (1975, p. 280). CAT proposes such a mechanism: stimuli uncorrelated with reinforcement are said to generate a classically condi- 
tioned response of inattention. Thus, classical conditioning and the laws governing the acquisition of conditioned responses become the explanation for the attentional decrement with repeated stimulus preexposure.

The proposed conditioning approach to LI demands that any manipulation that enhances Pav lovian conditioning should, if applied to the preexposure phase of the LI paradigm, facilitate inattention and thus decrease subsequent learning to the preexposed stimulus. A number of predictions from this approach have found empirical support: (1) a second stimulus placed in a conditioning relationship to the preexposed stimulus, thus maintaining the attentional response to that stimulus, attenuates the decremental effects of preexposure (Lubow, Alek, \& Arzy, 1975; Lubow, Schnur, \& Rifkin, 1976; Szakmary, 1977); (2) the decremental effect of preexposure is a direct function of the intensity of the preexposed stimulus (Crowell \& Anderson, 1972; Schnur \& Lubow, 1976); and (3) the intertrial interval during preexposure (Lantz, 1973; Schnur \& Lubow, 1976).

While the rate of conditioning to a given stimulus is a function of the characteristics of that stimulus, such as its intensity, as well as the subject's previous experience with it, it also depends upon whether the stimulus is presented alone or in a compound. When a compound CS, consisting of two elements, is conditioned, and each element is tested in isolation, the elements often exhibit different levels of conditioning. This difference in conditionability has been found to depend on the relative salience of the elements (Kamin, 1969). In addition, the more salient element of the compound "overshadows" the less salient one; that is, the latter exhibits poorer conditioning when conditioned in a compound as compared with when it is conditioned in isolation.

If conditioning of inattention occurs as postulated, then nonreinforced preexposure of a compound consisting of two elements of different salience should provide results parallel to those found in conventional compound conditioning. First, both the compound and its elements will show an increase of LI as a function of number of preexposures. Second, preexposure should produce different levels of latent inhibition for the two elements of the compound. Specifically, according to CAT, more latent inhibition should accrue to the more salient element of the compound than to the less salient element. Third, with the preexposure of a compound stimulus containing elements of different salience, one should be able to demonstrate overshadowing of LI to the less salient element.

To test the above predictions, we performed two experiments. Experiment 1 preexposed a compound consisting of two elements of different salience and tested for the amount of latent inhibition to each of the elements and the compound as a function of the number of preexposures. It was predicted that, as a result of nonreinforced preexposure of the compound, a greater loss of conditionability would accrue to the more conditionable element of the compound than to the less conditionable element. Experiment 2 provided a direct test for overshadowing during nonreinforced preexposure.

\section{EXPERIMENT 1}

According to CAT, the greater the conditionability of a stimulus when followed by a US, the greater is the conditioning of inattention to that stimulus when not followed by reinforcement. If, then, a compound stimulus, made up of two elements of differential salience, is preexposed, there should be more conditioned inattention accruing to the more salient element than to the less salient element; that is, the latent inhibition effect should be greater for the more salient than for the less salient element.

\section{Method}

Subjects. Forty-eight male albino Charles River rats were obtained from A. Loebenstein Animal Laboratories. At the start of the experiment, the animals were approximately 70 days of age. They were housed one to a cage under a 12 -h light-dark cycle with conditions of relatively constant temperature $\left(23^{\circ}-25^{\circ} \mathrm{C}\right)$ and humidity $(70 \%-86 \%$ relative humidity). All testing was done during the light portion of the cycle.

Apparatus. The apparatus consisted of a Perspex test chamber, $22 \times 19 \times 29 \mathrm{~cm}$ as measured from the raised grid floor. The floor was composed of $.30-\mathrm{cm}$ stainless steel bars with a between-bar separation of $1.5 \mathrm{~cm}$. A hole, $5 \mathrm{~cm}$ in diameter and $2.5 \mathrm{~cm}$ from the floor, was centered on one of the narrower panels. A water nozzle, extending $1 \mathrm{~cm}$ into the chamber, could be introduced through this hole. Water delivery was controlled by a solenoid valve. The dependent measure, number of licking responses, was detected by a standard drinkometer circuit. During the period when the nozzle was not available to the rat, the hole was covered with a Perspex lid. Shock was delivered through a Lafayette unscrambled shock source (Model A-615A) set at .4 mA.

The tone stimulus, a $2.8-\mathrm{kHz}$ signal, was produced by an EICO audio-oscillator (Model 377), the output of which went from a step-down transformer to an $8-\Omega$ speaker. The speaker was located $20 \mathrm{~cm}$ above the nozzle. Tone intensity, $77.5 \mathrm{~dB}$ SPL, was measured from the center of the grid floor. From the same point, the ambient masking noise produced by a ventilating fan was $37.5 \mathrm{~dB}$ at $2 \mathrm{kHz}$ octave (91 dB linear scale). The light stimulus was produced by a $3-\mathrm{W} 12-\mathrm{V}$ bulb located $10 \mathrm{~cm}$ above the nozzle. The light was diffused through an orange-colored cap. The chamber was placed in a sound-insulated chest. Stimulus presentations and schedules of reinforcement were controlled by a tape programmer, and the number of licks was recorded with a printout counter.

Procedure. On each of Days 1 and 2, the rats were allowed access to a water-filled Richter tube for $10 \mathrm{~min}$ in their home cages. On Day 3 , the animals were placed in the chamber for $40 \mathrm{~min}$. During this time, water was made available on a variableinterval (VI) 20-sec schedule. Each reinforcement consisted of a .1-cc drop of water. The VI schedule was maintained throughout the experiment except as indicated. After each session, the rat was returned to its home cage and allowed 10 min of access to a waterfilled Richter tube. Food was available in the home cage on an ad-lib basis. 
On Day 4, the rats were assigned randomly to four major groups: zero preexposure $(0)$, and 20,40 , and 80 preexposures to the tone-light compound (20-TL, 40-TL, 80-TL). On Days 4 and 5, each rat was placed in the chamber for a $40-\mathrm{min}$ period. The $80-\mathrm{TL}$ group received 40 preexposures to the compound on Day 4 and 40 on Day 5. The 40-TL group received all of its preexposures on Day 5. The 20-TL group received all of its preexposures on Day 5 , starting $20 \mathrm{~min}$ after the $40-\mathrm{min}$ session had begun. The preexposure treatments were superimposed on the VI 20-sec drinking schedule. The duration of the preexposed TL compound was $20 \mathrm{sec}$. The time between compound presentations averaged $60 \mathrm{sec}$ $( \pm 30 \mathrm{sec})$.

On Day 6, the water nozzle was removed from the test chamber and each rat was given four forward classical conditioning trials to the TL compound. After the rat had spent $2 \mathrm{~min}$ in the chamber, TL was presented for $20 \mathrm{sec}$. The last $.75 \mathrm{sec}$ of the CS coincided with the .4-mA shock. The intertrial interval was $2 \mathrm{~min}$. Two minutes after the termination of the fourth TL-shock presentation, the rat was removed from the test chamber and the drinking nozzle was reintroduced to the chamber. Immediately following this, the rat was returned to the chamber. After $10 \mathrm{~min}$, the test phase was initiated.

For the test phase, every subject received two successive presentations of each of the two elements of the compound and of the compound itself. Each presentation was $20 \mathrm{sec}$ in duration and was not followed by shock. The time between stimulus presentations was $280 \mathrm{sec}$. The order of test stimulus presentations was fully counterbalanced across subjects. The experiment was performed in six successive replications over a period of 6 weeks. Within each replication, two rats were assigned randomly to each of the four groups.

Suppression ratios, $B /(A+B)$, were calculated for each of the six test trials. $B$ is the number of licks during the $20-\mathrm{sec}$ tone, and $A$ is the number of licks during the preceding $20 \mathrm{sec}$ in the absence of the tone. A suppression ratio of 0 indicates a complete lack of responding during the $C S$ presentation, while a ratio of .50 indicates no change in response rate from the pre-CS period to the CS period.

\section{Results}

The six consecutive A periods for the four groups were examined using a $4 \times 6$ ANOVA. The groups did not differ in number of responses during the $A$ period $[F(3,44)=1.48, p>.10]$. Likewise, there was no reliable difference among the trials $[F(5,220)$ $<1]$. Thus, there is no evidence for nonspecific effects of the treatment variable or for sampling bias.

The suppression ratios for the four groups, to each of the three stimulus conditions, are shown in Figure 1. It is quite clear that as the number of preexposures to the TL compound, prior to its being paired with shock, increases, the amount of suppression decreases. This is substantiated by a $4 \times 3 \times 2$ ANOVA in which the first factor is the different number of preexposures, $0,20,40,80$; the second factor is the test stimulus, T, L, and TL, repeated; and the third factor is the two test trials, Trials 1 and 2 , for each test stimulus, repeated. The main effect of number of preexposures was highly significant $[F(3,44)=31.47, p<.001]$. Except for the difference between Groups 40-TL and 80-TL, all differences among the four preexposure groups were significant (Newman-Keuls tests, $p<.05$ ).

As can be seen from Figure 1, there were also differences as a function of test stimulus and an inter-

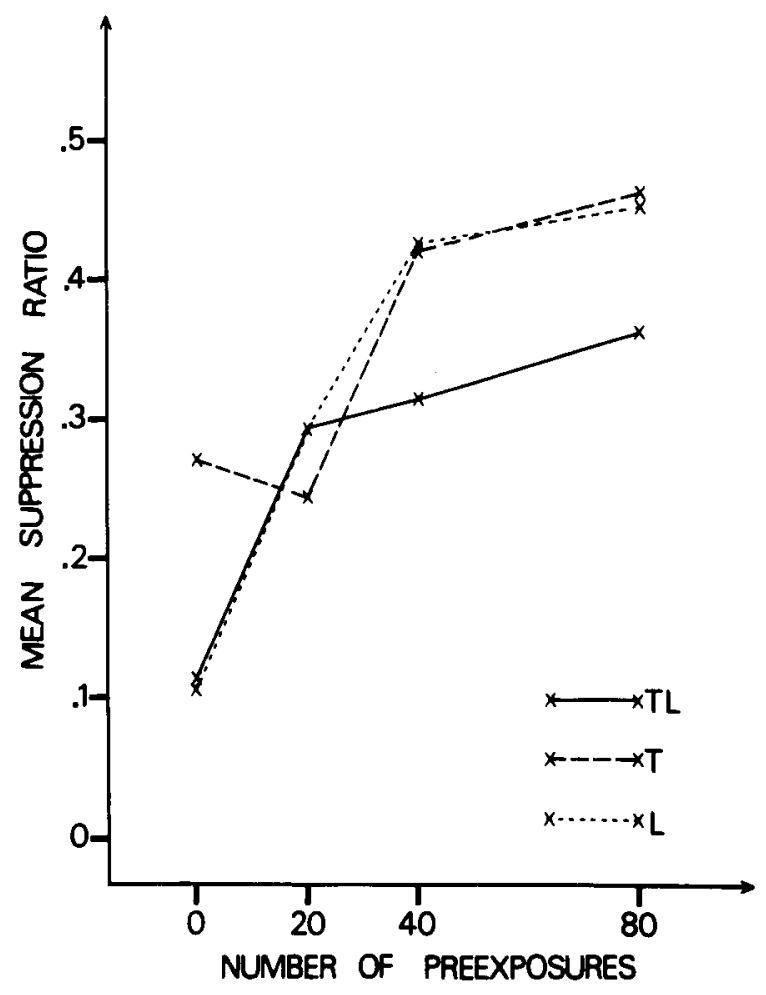

Figure 1. Mean suppression ratios as a function of number of preexposures.

action between test stimulus and number of preexposures; both were reliable $[F(2,88)=6.42, p<.01$; $F(6,88)=3.38, p<.01$, respectively]. These effects are accounted for by the fact that for the 0 group there was significantly more suppression to $L$ and $T L$ than to $T$ (Newman-Keuls test, $p<.05$ ), while for 20-TL there were no significant differences among the T, L, and TL test conditions (Newman-Keuls test, $p>.10$ ). The pattern of suppression to the test stimuli within Groups 40-TL and 80-TL was similar; suppression to $\mathrm{T}$ and to $\mathrm{L}$ did not differ but was significantly less than that to TL (Newman-Keuls test, $\mathrm{p}<.05$ ).

Additional Newman-Keuls tests indicated that the decline in suppression from 0 to 20-TL was significant for $L$ and TL $(p<.05)$ but not for T. From 20-TL to 40-TL, the decline in suppression was significant for $T$ and $L(p<.05)$ but not for TL. None of the three stimulus conditions showed a significant change in suppression ratio from 40-TL to 80-TL. The trials effect and all of its interactions were not significant.

\section{Discussion}

The data exhibit several noteworthy features. First of all, the results obtained in the 0-preexposure group clearly established that, for the particular tone and light used in this experiment, the light was more salient than the tone. In fact, conditioning to $\mathrm{L}$ while in the TL compound provided most of the effective 
conditioning strength of the compound. The T component of the TL compound added very little to the effectiveness of conditioning to TL. Thus, two stimuli were identified which, when conditioned in a compound, differed in salience.

Second, preexposure of the compound retarded the course of conditioning to the compound. This once again demonstrates latent inhibition within the conditioned suppression paradigm (e.g., Lubow \& Siebert, 1967; Rudy, Krauter, \& Gaffuri, 1976), but with a compound CS. In addition, the LI effect was shown to be a positive function of the number of preexposures (cf. Lubow, 1973, Table 2). The basic LI effect and the increase in $\mathrm{LI}$ as a function of number of preexposures was shown for each of the elements of the compound, as well as the compound itself.

Third, the main prediction of the experiment, that is, a greater amount of LI to the more salient light than to the tone, was confirmed, at least with a low number of preexposures. According to CAT, one would predict an increase in the control of $T$ (less $L I)$, as compared with $L$, over suppression as a function of the number of TL preexposures. This prediction is derived as follows: In the TL compound, $\mathrm{L}$ is more salient than $T$. Thus, with repeated preexposures to TL, there would be greater conditioning of inattention to $\mathrm{L}$ than to $\mathrm{T}$. This would allow increasingly favorable conditions for improving conditioning to the T element of the compound when TL was paired with shock in the acquisition phase. This, of course, would be reflected in more suppression to $\mathrm{T}$ than to $\mathrm{L}$ during the test phase. It is quite clear from Figure 1 that this is not the overall picture. $T$ and $L$ both end up with poor control over suppression. However, the number of preexposures to the compound has the predicted effect, but only after relatively few preexposures: $L$ loses control very rapidly in going from 0 to 20 preexposures (suppression ratios of .10 and .30 , respectively); $T$, on the other hand, does not lose control of suppression in going from 0 to 20 preexposures $(.27$ and .25 , respectively). Thus, the prediction regarding differences in amount of $\mathrm{LI}$ accruing to the two elements of the compound-light and tone-as a result of preexposure was confirmed. Despite very different suppression ratios for $\mathrm{T}$ and $\mathrm{L}$ after zero preexposures, with more conditioned suppression to $\mathrm{L}$ than to $\mathrm{T}$, the two elements reached the same level after 20 preexposures. It can be concluded that when two stimuli of different salience are preexposed in compound, more LI accrues to the more salient element of the compound. This conclusion is consistent with the findings of Schnur and Lubow (1976). In that experiment, in which different tone intensities were administered to different groups, preexposure to the more salient, high-intensity tone produced more LI than preexposure to the less salient, low-intensity tone.

It should be pointed out that there is a fundamental paradox involved in testing the proposition that more latent inhibition will accrue to the more conditionable stimulus. Since the amount of latent inhibition is defined as the difference between a zeropreexposed group and an n-preexposed group in a conditioning test, the more poorly conditionable stimulus after zero preexposures, by definition, starts at a level closer to the maximum LI effect than the more easily conditioned stimulus; therefore, if both arrive at the same conditioning level, there will be differences in $\mathrm{LI}$ as a result of the differences in starting point. This is seen quite clearly in Figure 1 and, of course, accounts for the data which support the prediction from CAT. This type of ceiling effect might be avoided with a more sensitive design which, as a function of the number of preexposures, would allow the more highly conditionable stimulus to reach the asymptote of $L I$ at a faster rate than the more poorly conditionable stimulus.

In addition to the differences in rates of accrual of LI to the light and the tone with relatively low numbers of preexposures, and the disappearance of the behavioral difference to the light and the tone with an increase in the number of preexposures, there is a change in the relative control of suppression by the compound and the elements. Although, with few preexposures, suppression is controlled most effectively by light, with a high number of preexposures suppression is controlled by the compound and not by the originally more salient light. This change was not predicted, since, given the design employed in the experiment, in which all subjects receive two pairings of the TL compound with shock, it was not possible to make a meaningful comparison of the amount of LI to the compound and the amount of LI to the elements. In other words, the measurable amount of LI to the compound in test is reduced by the fact that it was the TL compound that was paired with shock in acquisition. Therefore, there is a necessary underestimation of $\mathrm{LI}$ to the compound, and there is no way to validly compare LI to the compound and LI to the elements. However, the empirical finding that the preexposure of the compound results in relative changes of stimulus control is rather interesting. These changes suggest that the organism learns to discriminate between the elements and the compound as a function of repeated presentations of the compound.

With few exceptions (e.g., Lubow, Rifkin, \& Alek, 1976), simple, nonreinforced stimulus preexposure has been considered to generate a "negative" learning process: loss of subsequent associability to the preexposed stimulus. The present results also suggest that there is a "positive" learning process which takes place during nonreinforced preexposure: learning to discriminate between the preexposed stimulus elements and the compound in which they are embedded.

Returning to the major focus of Experiment 1, that is, the comparison between the amount of LI to 
the $\mathrm{T}$ and $\mathrm{L}$ elements of the TL compound, we note that the results obtained support the prediction derived from CAT, that preexposure to the more salient stimulus produces more LI than does preexposure to the less salient stimulus of a compound. CAT would further predict that the more salient element should overshadow the less salient one during compound preexposure.

To test this prediction, one should compare the amount of LI for the individually preexposed elements as well as for the compound. This latter test provides the necessary control groups for measuring overshadowing directly, rather than by inference. There should be appreciably more LI to $\mathrm{T}$ when $\mathrm{T}$ is preexposed alone than when $T$ is preexposed as part of the TL compound, particularly with a low number of preexposures. Experiment 2 was designed to test for the appearance of an overshadowing effect during preexposure.

\section{EXPERIMENT 2}

If the CAT explanation of the data from Experiment 1 was correct, then one would predict that nonreinforced preexposure of the elements would give the same pattern of results as preexposure to the compound-namely, more LI to the more salient $\mathrm{L}$ than to the less salient $T$. Furthermore, a comparison of the amount of $\mathrm{LI}$ accruing to $\mathrm{T}$ preexposed in a TL compound with $\mathrm{T}$ preexposed alone should show more LI in the latter case than in the former. This overshadowing effect would be predicted by CAT because any phenomenon demonstrable in normal classical conditioning should, under comparable conditions, be shown to be present during preexposure. Therefore, there should be less conditioned inattention (less LI) to the weakly salient tone when it is preexposed in compound with the strongly salient light than when it is preexposed by itself.

Experiment 2 used a $4 \times 2 \times 3$ mixed factorial design with four types of preexposure (no preexposure, light preexposure, tone preexposure, or tone-light compound preexposure). Each group was divided in half and tested on either the tone or the light. All eight groups received three blocks of two extinction trials.

\section{Method}

Subjects and Apparatus. The subjects were $\mathbf{4 0}$ male albino rats, Charles River strain, obtained from A. Loebenstein Animal Laboratories. They were approximately 70 days of age at the start of the experiment. Housing conditions, apparatus, and stimuli were the same as those of Experiment 1.

Procedure. On each of Days 1 and 2, the rats were allowed 10 min of access to a water-filled Richter tube in their home cages. On Days 3 and 4 , the subjects were placed in the experimental chamber for $\mathbf{4 0}$ min with water available from a nozzle on a VI 20-sec schedule. Each reinforcement consisted of a .1-cc drop of water. The rats were then divided randomly into four experimental groups. On Day 5, the three preexposure groups-tone preexposure (T-PE), light preexposure (L-PE), and tone-light compound preexposure (TL-PE)-each received their respective preexposure treatments superimposed on the VI 20-sec drinking schedule. All stimulus presentations were $20 \mathrm{sec}$ in duration. For all groups, the first preexposure occurred $60 \mathrm{sec}$ after the start of the session. Subsequent stimulus presentations were on a variable time schedule of $60 \mathrm{sec}( \pm 30 \mathrm{sec})$. Forty stimulus preexposures were presented to each rat. $\mathbf{A}$ fourth group received no stimulus preexposure (0-PE). In all other respects, the 0-PE group was treated like the stimulus preexposure groups.

On Day 6, all subjects were given four forward classical conditioning trials to the TL compound. The procedure was identical to that used in Experiment 1. Following the conditioning trials, the rats were placed in their home cages for $10 \mathrm{~min}$, after which they were returned to the experimental chamber for the test phase. Half of each group was tested with light, and the other half was tested with tone. The test phase consisted of six nonreinforced presentations of the appropriate stimulus. The stimulus presentations were superimposed on the VI 20-sec drinking schedule. Suppression ratios were calculated in the same manner as in Experiment 1 .

\section{Results}

The number of licking responses during six consecutive A periods for the eight groups were examined with an $8 \times 6$ ANOVA. There were no reliable differences among the groups during the $A$ period $[F(7,32)<1]$. Likewise, there was no reliable difference among trials $[F(5,160)=1.81, p>.10]$. As in Experiment 1, there was no evidence for nonspecific effects of the treatment variable or for sampling bias.

The mean suppression ratios for the six test trials for the eight groups are presented in Table 1 . A $4 \times 2 \times 3$ ANOVA (preexposed stimulus $\times$ test stimulus $\times$ blocks) was performed on the data. The effects of preexposed stimuli ( $T, L, T L$, or none) were significant $[F(3,32)=6.73, p<.01]$, as were the effects of the test stimuli (T or $L$ ) $[F(1,32)=26.91$, $\mathrm{p}<.001]$ and blocks $[\mathrm{F}(2,64)=82.19, \mathrm{p}<.001]$. The latter represents the expected decrease in suppression with continued testing under extinction conditions. However, there were no reliable interactions between blocks and any of the other variables. This indicates that the rate of change over the course of testing was similar for all of the groups. Most important, there was a significant interaction between preexposed stimuli and test stimuli. Basically, this reflects the LI phenomenon; there is a difference in the effect of the test stimulus, depending on whether or not it is preexposed. An inspection of Table 1 indicates that the group preexposed to light and tested on light showed

Table 1

Mean Suppression Ratios for All Groups (Experiment 2)

\begin{tabular}{lcc}
\hline & \multicolumn{2}{c}{ Test Condition } \\
\cline { 2 - 3 } $\begin{array}{c}\text { Preexposure } \\
\text { Condition }\end{array}$ & Tone & Light \\
\hline No Preexposure & .36 & .19 \\
Tone & .43 & .19 \\
Light & .31 & .38 \\
Tone + Light & .33 & .38 \\
\hline
\end{tabular}


less suppression (.38) than the group not preexposed and tested on light (.19). This difference, representing the amount of LI to light, is significant $(p<.05$, Newman-Keuls test). The presence of LI to light is likewise reflected in the comparison between the group preexposed to light and tested on light and the group preexposed to tone and tested on light (.38 vs. $.19 ; \mathrm{p}<.05)$. On the other hand, the tone preexposure group, when tested on tone, showed only marginally poorer suppression than the nonpreexposed group tested on tone (.43 vs. .36; $\mathrm{p}>.10)$. However, the presence of $\mathrm{LI}$ to tone is detected in the comparison between the groups preexposed to tone and tested on tone and the group preexposed to light and tested on tone (.43 vs. $.31 ; p<.05)$.

The amount of suppression to tone in the group preexposed to tone as compared with the group preexposed to the TL compound defines whether or not there is overshadowing. The difference between the two groups $(.43$ vs. .33$)$ is reliable $(p<.05)$, thus demonstrating the overshadowing effect. A comparable comparison for light, suppression to light for the L preexposed group as compared with the TL preexposed group, yields no significant difference $(.38$ vs. .38).

\section{Discussion}

The major results of Experiment 1 were replicated using a between-group design. First of all, the higher salience of $L$ than of $T$ was demonstrated by the greater suppression to $\mathrm{L}$ than to $\mathrm{T}$ for the 0-PE groups. Second, as in Experiment 1, more latent inhibition was demonstrated to $\mathrm{L}$ than to $\mathrm{T}$. Most importantly, the prediction of overshadowing of LI to the tone during preexposure was confirmed.

According to CAT, the accrual of conditioned inattention to the more salient $\mathrm{L}$ should interfere more with the accrual of conditioned inattention to $T$ during preexposure of TL than during preexposure of $T$ alone. This overshadowing would be reflected in greater suppression in the TL preexposed group tested on $T$ than in the $T$ preexposed group tested on $T$. This difference (.33 vs. .43) did occur in Experiment 2 and was statistically reliable.

That preexposure to $\mathrm{T}$ while embedded in the TL compound attenuates the $\mathrm{LI}$ effect to $\mathrm{T}$ as compared with preexposure of $\mathrm{T}$ alone has been reported elsewhere (Mackintosh, 1973; Rudy et al., 1976, Experiment 2, but not Experiment 1). However, these studies tested only one of the elements and the compound, and had no independent measure of element salience. The results of the present experiment suggest that this effect will hold true only for the less salient element, at least when the two stimulus elements are presented in a simultaneous temporal compound. When the two stimuli are presented in close temporal sequence, the attenuation of LI may not be limited to the less salient of the two stimuli (Lubow,
Alek, \& Arzy, 1975; Lubow, Schnur, \& Rifkin, 1976).

The fact that LI was again greater for $L$ than for $\mathrm{T}$ in this between-group design in which $\mathrm{T}$ and $\mathrm{L}$ were separately exposed to different groups weakens a generalization decrement hypothesis, at least if that hypothesis is meant to implicate the change in stimulus conditions from the preexposure phase to the test phase. In the present design, some of the groups were preexposed to the same element on which they were tested, as opposed to being preexposed to the compound and tested on an element. On the other hand, a generalization decrement may still occur from the acquisition phase (given to the compound for all groups) and the test phase (given to the element). However, whatever differences occur between the groups in the test phase cannot be attributed to the identical acquisition conditions, and thus must necessarily involve the differences between the groups during preexposure. This would appear logically to rule out the generalization decrement hypothesis as an explanation either for the LI effect itself or for the greater amount of LI to the more salient stimulus (L) than to the less salient stimulus ( $T$ ).

A more potent criticism of the designs of Experiments 1 and 2 concerns the locus of the effect of preexposure. The latent inhibition literature assumes that the effects of preexposure are primarily on the ability of the organism to form a new association with that stimulus. Thus, in the present experiments, it is assumed that the test phase difference reflects a difference in association strength accrued during the four CS-US acquisition trials. However, it is possible that the differential preexposure conditions directly affect behavior during testing; the same pattern of results would have been achieved if the acquisition session were eliminated from the procedure. The effect observed during testing would then simply be a result of differences in unconditioned response strength, which presumably should be different for different stimuli and wane as a function of number of preexposures. This possibility was not evaluated within the present designs. Although this possibly confounding factor remains a possibility, Lubow and Siebert (1969) have shown that the differences in unconditioned response strength as a function of the number of preexposures only partially accounts for the effects of CS preexposures on subsequent testing. Similarly, Domjan and Siegel (1971) showed that 5 stimulus presentations resulted in elimination of the unconditioned response to the stimulus, while 25 presentations were required to produce $L I$.

In summary, then, a number of predictions derived from CAT were tested and confirmed. Preexposure to a compound produced a latent inhibition effect to the compound and to its elements. This effect was a function of the number of preexposures. Preexposure to the more salient stimulus produced more LI 
than preexposure to the less salient stimulus. This occurred regardless of whether preexposure was to the elements as part of a compound or to the elements individually. Finally, the more salient stimulus overshadowed the less salient stimulus during compound preexposure. These findings offer considerable support for CAT as a model for explaining the effects of stimulus preexposure on subsequent learning.

\section{REFERENCES}

Crowell, C. R., \& Anderson, D. C. Variations in intensity, interstimulus interval and interval between preconditioning $C S$ exposure and conditioning with rats. Journal of Comparative and Physiological Psychology, 1972, 79, 291-298.

Domjan, M., \& Siegel, S. Conditioned suppression following CS preexposure. Psychonomic Science, 1971, 25, 11-12.

Kamin, L. S. Predictability, surprise, attention and conditioning. In B. A. Campbell \& R. M. Church (Eds.), Punishment and aversive behavior. New York: Appleton-Century-Crofts, 1969.

LANTZ, A. W. Effect of number of trials, interstimulus interval, and dishabituation during CS habituation on subsequent conditioning in a CER paradigm. Animal Learning \& Behavior, 1973, 1, 223-227.

Luвow, R. E. Latent inhibition. Psychological Bulletin, 1973, 79, 398-407.

Lubow, R. E., Aleк, M., \& Arzy, J. Behavioral decrement following stimulus preexposure: Effects of number of preexposures, presence of a second stimulus, and interstimulus interval in children and adults. Journal of Experimental Psychology: Animal Behavioral Processes, 1975, 1, 178-188.

lubow, R. E., Markman, R. E., \& Allen, J. Latent inhibition and classical conditioning of the rabbit pinna response. Journal of Comparative and Physiological Psychology, 1968, 66, 688-694.

Lubow, R. E., \& Moore, A. U. Latent inhibition: The effect of nonreinforced preexposure to the conditional stimulus. Journal of Comparative and Physiological Psychology, 1959, 52, 415-419.

Lubow, R. E., Rifkin, B., \& Alek, M. The context effect: The relationship between stimulus preexposure and environmental preexposure determines subsequent learning. Journal of Experimental Psychology: Animal Behavior Processes, 1976, 2, 38-47.

Lubow, R. E., Schnur, P., \& Rifkin, B. Latent inhibition and conditioned attention theory. Journal of Experimental Psychology: Animal Behavior Processes, 1976, 2, 163-174.
Lubow, R. E., \& Siebert, L. Latent inhibition within the CER paradigm. Journal of Comparative and Physiological Psychology, 1969, 68, 136-138.

Lubow, R. E., Weiner, I., \& Schnur, P. Conditioned attention theory. In G. H. Bower (Ed.), The psychology of learning and motivation (Vol. 15). New York: Academic Press, 1981.

Mackintosh, N. J. Stimulus selection: Learning to ignore stimuli that predict no change in reinforcement. In R. A. Hinde \& J. Stevenson-Hinde (Eds.), Constraints on learning. New York: Academic Press, 1973.

Mackintosh, N. J. A theory of attention: Variations in the associability of stimuli with reinforcement. Psychological Review, 1975, 82, 276-298.

Pearce, J. M., \& Hall, G. A model for Pavlovian learning: Variations in the effectiveness of conditioned but not unconditioned stimuli. Psychological Review, 1980, 87, 532-552.

RE1SS, S., \& WAGNER, A. R. CS habituation produces a "latent inhibition" effect but no active "conditioned inhibition." Learning and Motivation, 1972, 3, 237-245.

RESCORLA, R. A. Summation and retardation of latent inhibition. Journal of Comparative and Physiological Psychology, 1971, 75, 77-81.

Rescurla, R. A., \& Wagner, A. R. A theory of Pavlovian conditioning: Variations in the effectiveness of reinforcement and nonreinforcement. In A. H. Black \& W. F. Prokasy (Eds.), Classical conditioning II: Current research and theory. New York: Appleton-Century-Crofts, 1972.

Rudy, J. W., Krauter, E. E., \& Gaffuri, A. Attenuation of the latent inhibition effect by prior exposure to another stimulus. Journal of Experimental Psychology: Animal Behavior Processes, 1976, 2, 235-247.

Schnur, P. Selective attention: Effect of element preexposure on compound conditioning in rats. Journal of Comparative and Physiological Psychology, 1971, 76, 123-130.

Schnur, P., \& Lubow, R. E. Tests of the conditioned attention theory of latent inhibition: The effects of ITI and CS intensity during preexposure. Learning and Motivation, 1976, 7, 540-550.

Szakmary, G. A. A note regarding conditioned attention theory. Bulletin of the Psychonomic Society, 1977, 9, 142-144.

Wagner, A. Priming in STM: An information-processing mechanism for self-generated or retrieval-generated depression in performance. In T. J. Tighe \& R. N. Leaton (Eds.), Habituation: Perspectives from child development, animal behavior and neurophysiology. Hillsdale, N.J: Erlbaum, 1976.

Wolf, C., \& Maltzman, I. Conditioned orienting reflex and amount of preconditioning habituation. Proceedings of the American Psychological Association, 1968, 3, 129-130.

(Manuscript received December 9, 1981; revision accepted for publication February 9, 1982.) 